\title{
The bisphosphonate incadronate inhibits intraperitoneal dissemination in an in vivo pancreatic cancer model
}

\author{
SOICHI TAKIGUCHI ${ }^{1}$, YUKIKO NISHINO ${ }^{1}$, KAZUKO INOUE $^{1}$, MIYAKO IKEDA $^{3}$, YASUFUMI KATAOKA ${ }^{3}$, \\ KIMIHIKO MATSUSUE ${ }^{4}$, KENICHI NISHIYAMA ${ }^{2}$ and HARUO IGUCHI ${ }^{5}$ \\ ${ }^{1}$ Institute for Clinical Research and ${ }^{2}$ Department of Pathology, National Kyushu Cancer Center, Fukuoka 811-1395; \\ Departments of ${ }^{3}$ Pharmaceutical Care and Health Sciences and ${ }^{4}$ Hygiene Chemistry, Faculty of Pharmaceutical Sciences, \\ Fukuoka University, Fukuoka 814-0180; ${ }^{5}$ Clinical Research Institute, Shikoku Cancer Center, Matsuyama 791-0280, Japan
}

Received January 17, 2012; Accepted March 14, 2012

DOI: $10.3892 / o r .2012 .1757$

\begin{abstract}
Pancreatic cancer is characterized by intraperitoneal dissemination and often by large volumes of ascites. Aminobisphosphonates exhibit potent antitumor effects and are currently being tested against human solid tumors. Several aminobisphosphonates inhibit cancer cell migration by preventing the activation of Rho through inhibition of the mevalonate pathway. We evaluated the ability of an aminobisphosphonate, incadronate, to inhibit the growth of disseminated pancreatic cancer in vivo. We established an in vivo pancreatic cancer model with i.p. carcinomatosis in nude mice. Incadronate administration started from the day of tumor inoculation, and reduced tumor burden and ascites accumulation. Further, we evaluated the effect of incadronate on the inhibition of pancreatic cancer cell proliferation, migration and invasion in vitro. Incadronate induced growth inhibition and apoptotic death of pancreatic cancer cells. It also inhibited migration presumably by preventing the activation of Rho by lysophosphatidic acid. Thus, the in vivo antitumor effect may result from the inhibition of cancer cell proliferation and migration. The potent effects of incadronate in reducing tumor burden and ascites suggest that it will be of value in regimens for the treatment of pancreatic cancer.
\end{abstract}

\section{Introduction}

Pancreatic cancer is the fourth most common cause of cancer death in men and women in the United States, with a 5-year survival for all stages of the disease of less than $5 \%$ (1). Pancreatic cancer has no clear early warning signs or symptoms and is usually silent until the disease is well advanced. During the progression of pancreatic cancer, hepatic metastasis and peri-

Correspondence to: Dr Haruo Iguchi, Clinical Research Institute, Shikoku Cancer Center, Minamiumemotomachi Ko160, Matsuyama, Ehime 791-0280, Japan

E-mail: higuchi@shikoku-cc.go.jp

Key words: bisphosphonate, apoptosis, pancreatic cancer, intraperitoneal dissemination, Rho toneal dissemination are frequently seen as a distant metastasis, which results in a short survival period. Patients have a median survival of 4-8 months after diagnosis due to the advanced stage of disease by the time it has been discovered and treatment has begun. The anti-metabolite agent gemcitabine is currently being employed to treat pancreatic cancer (2). While gemcitabine has shown a significant benefit in clinical applications, its ability to impact pancreatic cancer is limited. Therefore, new therapeutic approaches need to be investigated to improve the treatment of this neoplasm (3).

One new therapeutic strategy is to clarify the mechanism of metastasis of cancer cells and to identify agents that prevent cancer cells from invading or migrating into the peritoneum. Among many growth-promoting factors known to be present in pancreatic cancer ascites, lysophosphatidic acid (LPA) is found in significant levels and may play an important role in the development or progression of ovarian $(4,5)$ and pancreatic cancer (6). LPA has been reported to induce many cellular effects, including mitogenesis, the secretion of proteolytic enzymes, and migration activity (7).

Cell migration is regulated by a combination of different processes: the contraction of actomyosin, the formation of stress fibers, and the turnover of focal adhesions (8). Contraction of the actomyosin system is important for cell migration, and LPA induces myosin light-chain phosphorylation through the activation of the small GTP-binding protein ( $G$ protein) Rho, leading to the stimulation of cell contractility and motility (9). Another fundamental component affecting cell motility is focal adhesions (cell-extracellular matrix adhesions) (8). Rho is a key mediator in the assembly of structures involved in focal adhesion. Changes in the expression and activities of the components of focal adhesions may contribute to cancer invasion (10). Interfering with the LPA signal transduction pathway by modulating Rho activity may be an attractive strategy for improving the outcome of pancreatic cancer.

Aminobisphosphonates (N-BPs) are potent inhibitors of bone resorption used for the treatment and prevention of osteoporosis. N-BPs have been shown to inhibit the cholesterol biosynthesis pathway as well as isoprenylation (farnesylation and geranylgeranylation) by inhibiting either isopentanyl diphosphate synthase or a downstream enzyme, farnesyl diphosphate synthase, or both (11). Protein targets of isoprenylation include small $\mathrm{G}$ proteins 
such as Rho, Ras, Rac, and Rab, which require post-translational modification to undergo a series of changes that lead to their attachment to the plasma membrane and their full activation. The activation of small $\mathrm{G}$ proteins is essential for cancer cell growth and invasion (12,13). Accordingly, N-BPs have the potential to inactivate the small $\mathrm{G}$ proteins that regulate cancer cell growth, motility, and invasion $(14,15)$. Treatment of ovarian cancer cells with alendronate, an N-BP, resulted in the inactivation of Rho, changes in cell morphology, loss of stress fiber formation, and focal adhesion assembly (16). Furthermore alendronate markedly inhibited the invasiveness of human ovarian cancer cells in a model of i.p. ovarian carcinomatosis (17).

In the present study, we evaluated the ability of incadronate (INC), an N-BP, to inhibit the growth of disseminated pancreatic cancer in vivo. We established an in vivo pancreatic cancer model with i.p. carcinomatosis in nude mice. INC administration started from the day of tumor inoculation, and reduced tumor burden and ascites accumulation. Additionally, we evaluated the effect of INC on the inhibition of pancreatic cancer cell proliferation, migration, and invasion in vitro.

\section{Materials and methods}

Cell culture. SUIT-2, AsPC-1, and BxPC-3 cell lines were derived from a human pancreatic ductal adenocarcinoma (PDAC). They were cultured in a RPMI-1640 medium (Invitrogen, Carlsbad, CA, USA) supplemented with $5 \%$ fetal bovine serum (FBS) and $50 \mathrm{U} / \mathrm{ml}$ penicillin and $50 \mu \mathrm{g} / \mathrm{ml}$ streptomycin in a humidified atmosphere under $5 \% \mathrm{CO}_{2}$ at $37^{\circ} \mathrm{C}$.

In vitro cell proliferation assay. Cell proliferation was measured using an MTT dye reduction method (18). Briefly, human PDAC cell lines were seeded into 96 -well plates $\left(5 \times 10^{3}\right.$ cells $\left./ 100 \mu \mathrm{l}\right)$ and allowed to attach for $24 \mathrm{~h}$. Cells were treated with various concentrations of INC (Astellas Pharma, Tokyo, Japan) for $48 \mathrm{~h}$ in $100 \mu \mathrm{l}$ culture medium. At the end of drug exposure, $5 \mu \mathrm{l}$ MTT stock solution ( $5 \mathrm{mg} / \mathrm{ml}$ PBS) was added to each well, and the cells were further incubated for $4 \mathrm{~h}$ at $37^{\circ} \mathrm{C}$. Then, $100 \mu 1$ of DMSO was added to dissolve the dark blue crystals. Absorbance was measured with an MPR-A4 microplate reader (Tosoh, Tokyo, Japan) at test and reference wavelengths of 540 and $620 \mathrm{~nm}$, respectively.

Analysis of apoptosis. SUIT-2 cells were cultured in slide chambers to no more than 50-60\% confluence and treated with various concentrations of INC, as described in in vitro cell proliferation assay. After 48-h treatment, apoptosis was assessed using an in situ cell death detection kit (Roche Applied Science, Mannheim, Germany), following the manufacturer's recommendations. Briefly, cells were washed with PBS once, fixed with $4 \%$ buffered paraformaldehyde for $1 \mathrm{~h}$, washed again with PBS, and permeabilized on ice with $0.1 \%$ Triton $\mathrm{X}-100$ in $0.1 \%$ sodium citrate for $2 \mathrm{~min}$. Slides were rinsed twice with PBS and then incubated for $60 \mathrm{~min}$ at $37^{\circ} \mathrm{C}$ with terminal deoxynucleotidyl transferase enzyme in reaction buffer. The slides were rinsed three times with PBS and mounted with PermaFluor (Thermo Fisher Scientific, Waltham, MA, USA). Samples were analyzed by fluorescence microscopy. TUNEL-positive nuclei were detected by a bright color in condensed or ruptured nuclei. The rate of apoptosis was calculated as the ratio of the number of apoptotic cells to the total number of cells (both apoptotic and non-apoptotic cells).

Cell migration and invasion assays. Transwell cell migration and invasion were evaluated using a 24-well chemotaxis chamber with a membrane of $8 \mu \mathrm{m}$ pore size (BD Biosciences, Franklin Lakes, NJ, USA). For migration and invasion assays, SUIT-2 cells were incubated in serum-free culture medium with the various concentrations of INC for $24 \mathrm{~h}$ and transferred to the upper chamber $\left(2.5 \times 10^{5}\right.$ cells $\left./ 500 \mu \mathrm{l}\right)$ with the various concentrations of INC and allowed to migrate through control (non-coated) and Matrigel-coated $(8.7 \mathrm{mg} / \mathrm{ml})$ membranes for $24 \mathrm{~h}$, respectively. The lower chambers were filled with culture medium containing $5 \%$ FBS with the various concentrations of INC. Non-migrated cells were wiped off with a cotton swab, the filter was stained with Diff-Quik stain solution (Siemens, Munch, Germany), and the number of remaining cells was counted under the microscope. To determine the percent of invasion, the mean number of cells invading through the Matrigel-coated insert membrane was divided by the mean number of cells migrating through the control insert membrane and multiplied by 100 .

Rho pull-down assay. The Rho pull-down assay was performed using a Rho activation assay kit according to the manufacturer's instructions (Cytoskeleton, Denver, CO, USA). Briefly, cells $\left(3 \times 10^{5} / \mathrm{ml}\right)$ were cultured under serum-free conditions with or without various agents for $24 \mathrm{~h}$. After incubation, the cells were stimulated with 2.2 $\mu \mathrm{M}$ LPA for 1 min and lysed in $\mathrm{Mg}^{2+}$ lysis buffer. Equal volumes of cell lysates were incubated with Rhotekin-RBD beads. Bound Rho proteins were detected by Western blotting using a monoclonal antibody against RhoA. Western blotting of the total amount of Rho in cell lysates was performed for comparison with Rho activity (level of GTP-bound Rho) in the same samples.

In vivo peritoneal dissemination model of pancreatic cancer. Five-week-old male nude mice (BALB-cAJcl-nu/nu, Clea Japan, Tokyo, Japan) were housed in filtered-air laminar-flow cabinets and were manipulated using aseptic procedures. Procedures involving animals and their care were conducted in conformity with the guidelines of the National Kyushu Cancer Center. To prepare the in vivo peritoneal dissemination model, SUIT-2 cells were injected i.p. as a cell suspension into nude mice (1x10 ${ }^{6}$ cells in $200 \mu 1$ PBS per animal). This model using SUIT-2 is not only simple and reproducible, but also has characteristics that resemble those of human pancreatic cancer. The treatment regimens started on the day of tumor inoculation and continued for 4 weeks. INC was delivered using a PBS vehicle and was administered i.p. every day. The daily doses of INC used were as follows: $0,0.1,1,2$, and $3 \mathrm{mg} / \mathrm{kg} / \mathrm{d}$. At the end of the treatment period, mice were sacrificed. The volume of ascites was measured, and tumor tissue was excised, weighed, fixed in $10 \%$ neutral buffered formalin, and embedded in paraffin. Paraffin sections $(5 \mu \mathrm{m})$ were used for histological analysis. Sections were stained with hematoxylin and eosin, examined, and photographed under a microscope. Blood samples were collected from the left heart ventricle and assayed for serum CA19-9.

Statistical analysis. Results are reported as the average \pm SEM. Group comparisons were performed using a one-way analysis 
A

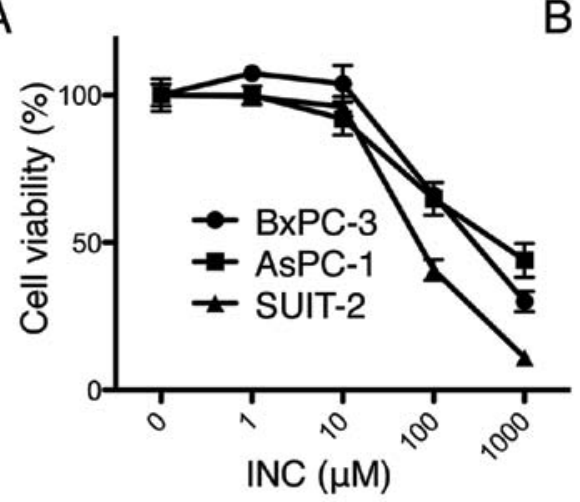

C
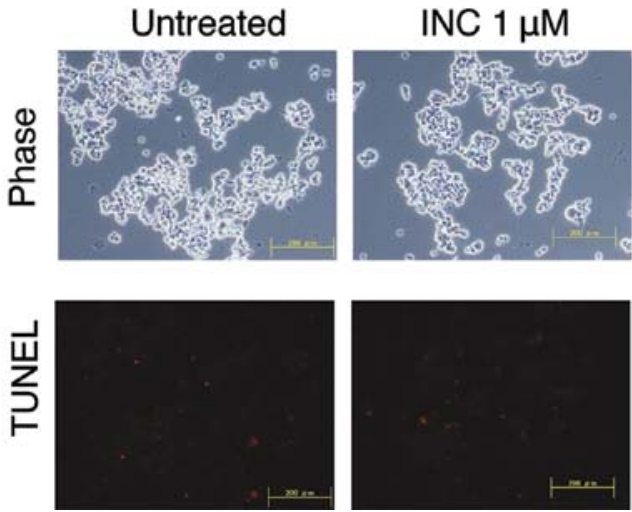

B

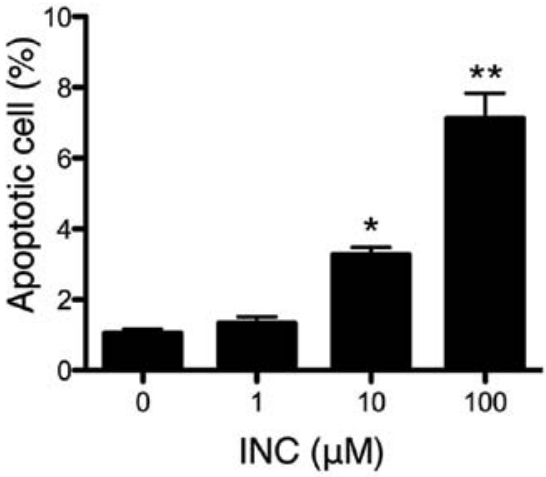

INC $(\mu \mathrm{M})$
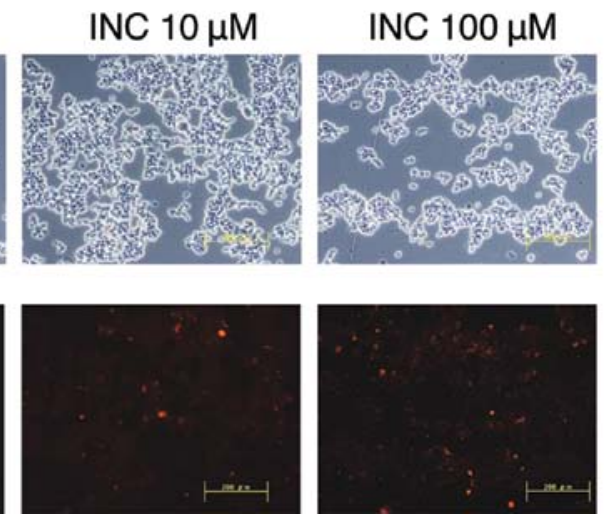

Figure 1. INC induces antiproliferative effects and apoptosis in human PDAC cell lines. (A) BxPC-3, AsPC-1, and SUIT-2 were treated with INC for 48 h. Cell growth inhibition was expressed as a percentage of the absorbance of control cultures measured at $540 \mathrm{~nm}$ with a microplate reader. $\mathrm{N}=6$ for each experiment. (B) The rate of apoptosis in SUIT-2 cells treated with INC for $48 \mathrm{~h}$ was calculated as the ratio of the number of apoptotic cells to the total number of cells. N=3 for each experiment. ${ }^{*} \mathrm{p}<0.05,{ }^{* *} \mathrm{p}<0.01$ vs. untreated cells. (C) Representative microscopic photos of SUIT-2 cells treated with INC for 48 h. Phase-contrast microscopy (upper panels) and fluorescent microscopy of TUNEL staining (white) showing apoptotic cells (lower panel). Bar, $200 \mu \mathrm{m}$.

of variance (ANOVA) followed by the Tukey-Kramer multiple comparison test. Differences were considered significant at $\mathrm{P}<0.05$.

\section{Results}

INC induces antiproliferative effects and apoptosis in human PDAC cell lines. The effect of INC on BxPC-3, AsPC-1, and SUIT-2 PDAC cell lines was investigated in vitro using the MTT assay. Treatment with INC (1-1000 $\mu \mathrm{M})$ produced a dose-dependent reduction in cell growth after $48 \mathrm{~h}$ of treatment (Fig. 1A) and the $\mathrm{IC}_{50}$ was calculated in a range of 50-500 $\mu \mathrm{M}$. To clarify the mechanisms of INC-induced growth inhibition, we performed a TUNEL assay on SUIT-2 cells, which were the most sensitive to this compound among the three PDAC cell lines tested. Thereafter, we used SUIT-2 cells exclusively in the other experiments. Upon 48-h exposure to INC, the occurrence of apoptosis in 10 and $100 \mu \mathrm{M}$ INC-treated cells was 3.3 and $7.1 \%$ respectively, and was significantly higher than that in untreated cells (1.1\%) (Fig. 1B). SUIT-2 cells presented typical apoptotic morphology with cell shrinkage, nuclear condensation and fragmentation, and cellular rupture into debris (Fig. 1C).

Effect of INC on migration and invasion of SUIT-2 in culture. INC was unable to perturb cellular migration toward 5\% FBS in chemotaxis chambers at $1 \mu \mathrm{M}$ (Fig. 2A). At 10 and $100 \mu \mathrm{M}$, the inhibition was 49 and $73 \%$, respectively. INC was also potent in its action against the invasion of cells through Matrigel with a $71 \%$ inhibition occurring at $10 \mu \mathrm{M}$ and a $90 \%$ inhibition at $100 \mu \mathrm{M}$ (Fig. 2B). These results are not due to cell cytotoxicity because the degree of cell death after 48-h treatment with INC did not exceed 5 and $10 \%$ at 10 and $100 \mu \mathrm{M}$ INC, respectively. The percent of invasion was 9.6, 10,5.5, and $3.7 \%$ at $0,1,10$, and $100 \mu \mathrm{M}$, respectively (Fig. 2C). Collectively, INC inhibited both migration and invasion of SUIT-2 at a concentration between 10 and $100 \mu \mathrm{M}$.

The activation of Rho by LPA is suppressed by INC, and restored by the addition of GGOH in SUIT-2. It is now well established that cell migration is induced by Ras-related GTPases (especially Rho). Soluble factors from serum, such as lysophosphatidic acid (LPA), are thought to activate Rho based on their ability to induce actin stress fibers and focal adhesions in a Rho-dependent manner (19). To evaluate whether LPA induces Rho activity in SUIT-2 cells, we used a pull-down assay with the fusion protein GST- Rhotekin-RBD, which recognizes only Rho-GTP, the active form of Rho. An increase in Rho-GTP was observed in SUIT- 2 cells treated for 1 min with LPA $(2.2 \mu \mathrm{M})$. The inhibitory effect of INC $(30 \mu \mathrm{M})$ and abrogation by the addition of GGOH $(30 \mu \mathrm{M})$ suggests INC may inhibit cancer cell migration through Rho geranylgeranylation (Fig. 3).

Effects of INC in a SUIT-2 peritoneal dissemination model. To assess the effect of INC on intraperitoneal dissemination in vivo, 

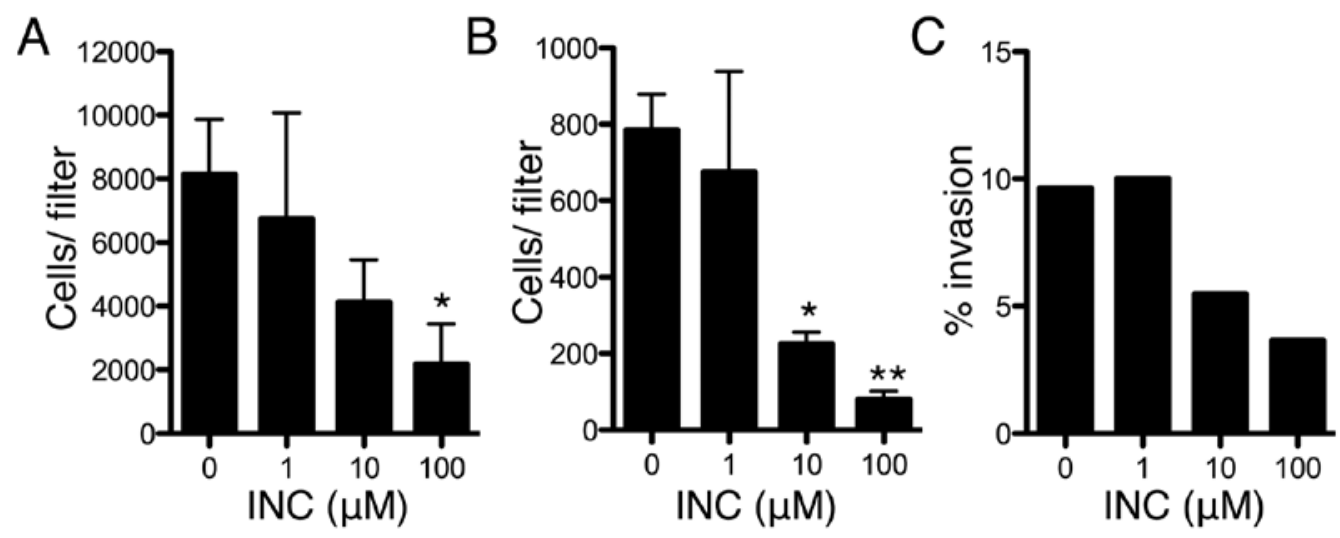

Figure 2. INC inhibits migration and invasion activities of SUIT-2. (A) For migration activity, SUIT-2 (2.5x10 $)$ cells were incubated with the indicated concentration of INC for $24 \mathrm{~h}$ and allowed to migrate through non-coated transwell plates for $24 \mathrm{~h}$. (B) For invasion activity, SUIT-2 (2.5x10 $)$ cells were incubated with the indicated concentration of INC for $24 \mathrm{~h}$ and allowed to migrate through Matrigel-coated $(8.7 \mathrm{mg} / \mathrm{ml})$ transwell plates for $24 \mathrm{~h}$. In either case, after fixing and staining with Diff-Quik stain solution, the number of migrated or invaded cells was counted. Bar, $200 \mu \mathrm{m}$. N=3 for each experiment. ${ }^{\mathrm{p}}<0.05$, $^{* *} \mathrm{p}<0.01$ vs. untreated cells.

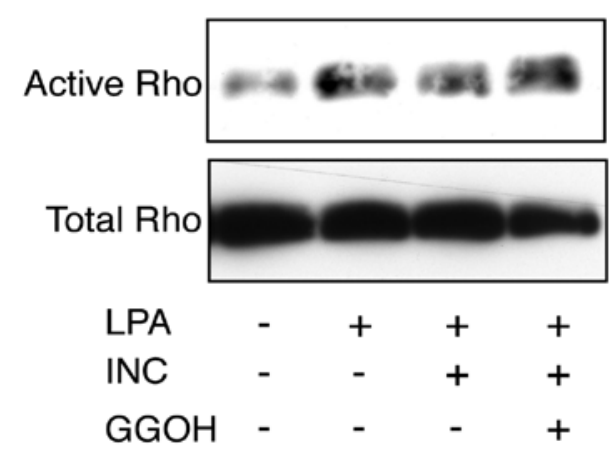

Figure 3. The activation of Rho by LPA is suppressed by INC, and restored by the addition of GGOH in SUIT-2. SUIT-2 cells were cultured under serum-free conditions with or without the various agents indicated for $24 \mathrm{~h}$. After incubation, the cells were stimulated with LPA. The cell lysates were incubated with Rhotekin-RBD beads. Bound Rho proteins were detected by Western blotting using a monoclonal antibody against RhoA (upper panel). Western blotting of the total amount of Rho in cell lysates (lower panel) was performed for comparison with Rho activity (level of GTP-bound Rho) in the same lysates. Blots are representative of three experiments. Relative amounts of active Rho normalized with the amounts of total Rho were determined by densitometric scanning and shown. The concentrations of agents that were used are as follows: LPA $2.2 \mu \mathrm{M}$; INC $30 \mu \mathrm{M}$; GGOH $30 \mu \mathrm{M}$.

we used a pancreatic cancer model with i.p. carcinomatosis in nude mice. We started the administration of INC on the day of cancer cell inoculation. Preliminary experiments revealed that tumor-bearing mice began to exhibit abdominal swelling with ascites $\sim 2.5$ weeks after cancer cell inoculation and died from cachexia after the 5 th week without any treatment. Therefore, we sacrificed and examined mice 4 weeks after cancer cell inoculation. At the autopsy examination, tumors were found on the surface of the peritoneum, diaphragm, intestines, liver, spleen, pancreas, and kidney, with massive ascites in the control group. The extent of tumor burden varied among the different treatment groups (Fig. 4A). Mean tumor weight in the peritonea of the untreated group was $1.58 \pm 0.29 \mathrm{~g}$, and was significantly reduced by $\sim 40 \%$ in the group given $1 \mathrm{mg} / \mathrm{kg} / \mathrm{d}(0.63 \pm 0.14 \mathrm{~g}$; $\mathrm{p}<0.05)$, by $\sim 40 \%$ in the group given $2 \mathrm{mg} / \mathrm{kg} / \mathrm{d}(0.63 \pm 0.15 \mathrm{~g}$; $\mathrm{p}<0.05)$, and by $\sim 39 \%$ in the group given $3 \mathrm{mg} / \mathrm{kg} / \mathrm{d}(0.62 \pm 0.13 \mathrm{~g}$; $\mathrm{p}<0.05$ ) (Fig. 4B). Fig. 4C shows the effects of INC treatment on ascites formation. The inhibitory effect of INC on ascites formation was dose-dependent. The mean volume of ascites in the untreated group was $2.91 \pm 0.26 \mathrm{ml}$, and was significantly reduced by $\sim 49 \%$ in the group given $1 \mathrm{mg} / \mathrm{kg} / \mathrm{d}(1.42 \pm 0.40 \mathrm{ml}$; $\mathrm{p}<0.05)$. Strikingly, INC treatment at $2 \mathrm{mg} / \mathrm{kg} / \mathrm{d}$ and $3 \mathrm{mg} / \mathrm{kg} / \mathrm{d}$ reduced ascites formation by $\sim 23 \%(0.67 \pm 0.49 \mathrm{ml} ; \mathrm{p}<0.01)$ and by $\sim 21 \%(0.60 \pm 0.28 \mathrm{ml} ; \mathrm{p}<0.01)$, respectively. The concentration of CA19-9, which was expressed from SUIT-2 (20), was examined in the serum collected from the left heart ventricle (Fig. 4D). The effect of INC treatment on the mean concentrations of CA19-9 was dose-dependent, although this did not reach statistical significance. Fig. 4E shows the histological appearance of the tumor nest formed after dissemination in the peritoneum from the untreated group. It shows how the tumor severely invaded into the peritoneum.

\section{Discussion}

Pancreatic cancer remains one of the most difficult malignancies to treat. Even patients who are eligible for curative surgical resection gain only moderate benefits. Furthermore, the lack of effective chemotherapeutic and targeted agents highlights the urgent need to better understand pancreatic cancer biology in order to guide the development of novel treatment strategies. During the progression of pancreatic carcinomas, cancer cells released from the surface of the tumor can adhere to and invade tissues and organs in the peritoneal cavity. Consequently, the poor outcome is, at least in part, due to peritoneal dissemination caused by the aggressive migration activity of pancreatic cancer cells $(21,22)$. To undergo peritoneal dissemination, pancreatic cancer cells need to detach from the primary tumor, attach to the cell-extracellular matrix of other tissues, and migrate into the stroma. Once cancer cells migrate into the stroma, angiogenesis occurs from pre-existing capillaries or venulae. Currently available anticancer drugs, even if they are very effective at killing cancer cells, can be used only at limited concentrations because of their toxicity to normal cells. Accordingly, it is worthwhile looking for drugs that inhibit the progression of pancreatic cancer affecting these properties, in addition to cell proliferation. 

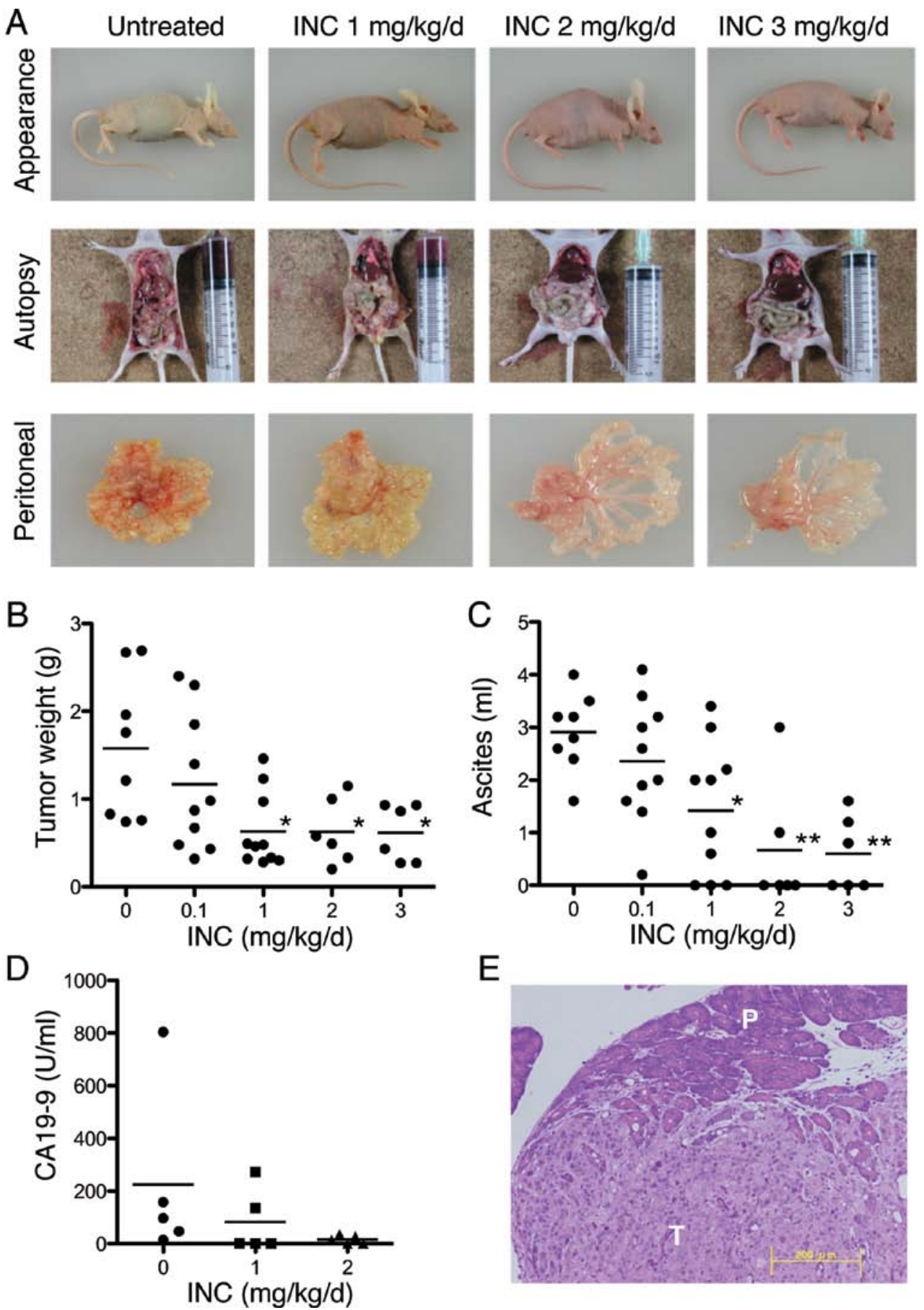

Figure 4. INC inhibits intraperitoneal dissemination in an in vivo pancreatic cancer model. (A) A SUIT-2 cell suspension (1x10 $/ 200 \mu 1 /$ mouse) was injected i.p. into nude mice and treated with the indicated amounts of INC everyday. Mice were sacrificed at 28 days after implantation and ascites, peritoneum, and blood sera were collected. The volumes of ascites (B), the weights of tumors disseminated on the peritoneum (C) and the concentrations of CA19-9 (D) were measured. $\mathrm{N}=6-10$ (B and C); $\mathrm{n}=5$ (D). ${ }^{*} \mathrm{p}<0.05,{ }^{* *} \mathrm{p}<0.01 \mathrm{vs}$. untreated mice. (E) Histologic appearance of the tumor nest formed after dissemination in the peritoneum from a SUIT-2-implanted nude mouse. Nude mice were injected i.p. with SUIT-2 cells, sacrificed at 28 days after implantation, and the peritonea were collected. Tissue was fixed, sectioned, and stained with hematoxylin and eosin. T, tumor nest; P, peritoneum, Bar, $200 \mu \mathrm{m}$.

Previous reports on the direct effects of N-BPs on cancer cells in vitro focused on cell viability and migration/invasion, and the effective concentrations of N-BPs were relatively high $(14,16,23,24)$. According to these reports, the concentrations of alendronate, ibandronate, pamidronate, and zoledronate that induced apoptosis and inhibited invasion of breast, prostate, and ovarian cancer cells were 10-100 $\mu \mathrm{M}$. The present study also demonstrated that the effective concentrations of INC on the induction of apoptosis and the inhibition of migration/inva- sion of pancreatic cancer cells were $10-100 \mu \mathrm{M}$, whereas the concentration of INC that significantly inhibits osteoclast-like cell formation in vitro is $0.01-0.1 \mu \mathrm{M}$, which is a clinically used concentration of INC (25). Although it is difficult to treat patients with N-BPs at concentrations of around 10-100 $\mu \mathrm{M}$ because of toxicity, clinical doses may be effective in the treatment of tumors for the following reasons. Tumor-associated macrophages (TAMs) play a pivotal role in tumor growth and metastasis by promoting tumor angiogenesis. Phagocytosis of N-BPs by TAMs 
could lead to its depletion by apoptosis and elevate the concentration of N-BPs around the tumor (26).

We evaluated the ability of INC to inhibit the progression of disseminated pancreatic cancer in vivo. To accomplish this, we established an in vivo peritoneal dissemination model of pancreatic cancer in nude mice. In this model, cancer cells are injected i.p., and, as such, do not reproduce the early events of peritoneal dissemination, i.e. cancer development and detachment from a primary tumor. However, our previous results from a SUIT-2 orthotopic implantation model were nearly identical to those from the i.p. injection model (27), so we decided to use this simple and reproducible model in the present study. Similar to our in vivo results from pancreatic cancer cells, the N-BP alendronate, markedly inhibited tumor growth, invasiveness, and ascites formation of human ovarian cancer cells in a model of i.p. ovarian carcinomatosis (17). Such in vivo antitumor effects by N-BPs may be derived from the suppression of cancer cell proliferation by apoptosis as well as migration/invasion through the inhibition of molecules in the mevalonate pathway, especially Rho, in the target cancer cells.

This is the first report showing that an N-BP inhibited pancreatic cancer cell invasion to visceral organs in vivo and may provide the basis for a new therapy to control the dissemination of pancreatic cancer.

\section{References}

1. Kleeff J, Michalski C, Friess H and Buchler MW: Pancreatic cancer: from bench to 5-year survival. Pancreas 33: 111-118, 2006.

2. Ueno H, Kiyosawa $\mathrm{K}$ and Kaniwa N: Pharmacogenomics of gemcitabine: can genetic studies lead to tailor-made therapy? Br J Cancer 97: 145-151, 2007.

3. Reni M, Cereda S and Galli L: PEFG (cisplatin, epirubicin, 5-fluorouracil, gemcitabine) for patients with advanced pancreatic cancer: the ghost regimen. Cancer Lett 256: 25-28, 2007.

4. Xu Y, Shen Z, Wiper DW, et al: Lysophosphatidic acid as a potential biomarker for ovarian and other gynecologic cancers. JAMA 280: 719-723, 1998.

5. Baker DL, Morrison P, Miller B, et al: Plasma lysophosphatidic acid concentration and ovarian cancer. JAMA 287: 3081-3082, 2002.

6. Yamada T, Sato K, Komachi M, et al: Lysophosphatidic acid (LPA) in malignant ascites stimulates motility of human pancreatic cancer cells through LPA1. J Biol Chem 279: 65956605, 2004.

7. Mills GB and Moolenaar WH: The emerging role of lysophosphatidic acid in cancer. Nat Rev Cancer 3: 582-591, 2003.

8. Horwitz AR and Parsons JT: Cell migration - movin' on. Science 286: 1102-1103, 1999.

9. Chrzanowska-Wodnicka M and Burridge K: Rho-stimulated contractility drives the formation of stress fibers and focal adhesions. J Cell Biol 133: 1403-1415, 1996.
10. Honn KV, Tang DG and Chen YQ: Platelets and cancer metastasis: more than an epiphenomenon. Semin Thromb Hemost 18 392-415, 1992.

11. Fisher JE, Rogers MJ, Halasy JM, et al: Alendronate mechanism of action: geranylgeraniol, an intermediate in the mevalonate pathway, prevents inhibition of osteoclast formation, bone resorption, and kinase activation in vitro. Proc Natl Acad Sci USA 96: 133-138, 1999.

12. Oxford G and Theodorescu D: Ras superfamily monomeric G proteins in carcinoma cell motility. Cancer Lett 189: 117-128, 2003.

13. Jaffe AB and Hall A: Rho GTPases in transformation and metastasis. Adv Cancer Res 84: 57-80, 2002.

14. Virtanen SS, Vaananen HK, Harkonen PL and Lakkakorpi PT: Alendronate inhibits invasion of PC-3 prostate cancer cells by affecting the mevalonate pathway. Cancer Res 62: 2708-2714, 2002.

15. Tassone $\mathrm{P}$, Tagliaferri $\mathrm{P}, \mathrm{V}$ iscomi $\mathrm{C}$, et al: Zoledronic acid induces antiproliferative and apoptotic effects in human pancreatic cancer cells in vitro. Br J Cancer 88: 1971-1978, 2003.

16. Sawada K, Morishige K, Tahara M, et al: Alendronate inhibits lysophosphatidic acid-induced migration of human ovarian cancer cells by attenuating the activation of rho. Cancer Res 62: 6015-6020, 2002.

17. Hashimoto K, Morishige K, Sawada K, et al: Alendronate inhibits intraperitoneal dissemination in in vivo ovarian cancer model. Cancer Res 65: 540-545, 2005.

18. Green LM, Reade JL and Ware CF: Rapid colorimetric assay for cell viability: application to the quantitation of cytotoxic and growth inhibitory lymphokines. J Immunol Methods 70: 257-268, 1984.

19. Ren XD, Kiosses WB and Schwartz MA: Regulation of the small GTP-binding protein Rho by cell adhesion and the cytoskeleton. EMBO J 18: 578-585, 1999.

20. Iwamura T, Katsuki T and Ide K: Establishment and characterization of a human pancreatic cancer cell line (SUIT-2) producing carcinoembryonic antigen and carbohydrate antigen 19-9. Jpn J Cancer Res 78: 54-62, 1987.

21. Vogel I, Kalthoff H, Henne-Bruns D and Kremer B: Detection and prognostic impact of disseminated tumor cells in pancreatic carcinoma. Pancreatology 2: 79-88, 2002.

22. Su D, Yamaguchi K and Tanaka M: The characteristics of disseminated tumor cells in pancreatic cancer: a black box needs to be explored. Pancreatology 5: 316-324, 2005.

23. Senaratne SG, Pirianov G, Mansi JL, Arnett TR and Colston KW: Bisphosphonates induce apoptosis in human breast cancer cell lines. Br J Cancer 82: 1459-1468, 2000.

24. Hiraga T, Williams PJ, Mundy GR and Yoneda T: The bisphosphonate ibandronate promotes apoptosis in MDA-MB-231 human breast cancer cells in bone metastases. Cancer Res 61: 4418-4424, 2001.

25. Kitagawa Y, Hiraga T, Yura Y and Yoneda T: Suppression by incadronate of invasion and growth of A-375 human melanoma in mandible in nude mice. Oncol Rep 13: 211-216, 2005.

26. Rogers TL and Holen I: Tumour macrophages as potential targets of bisphosphonates. J Transl Med 9: 177, 2011.

27. Iguchi H, Mizumoto K, Shono M, Kono A and Takiguchi S: Pancreatic cancer-derived cultured cells: Genetic alterations and application to an experimental model of pancreatic cancer metastasis. In: Culture of Human Tumor Cells. Pfragner R and Freshney RI (eds.) John Wiley \& Sons, Inc., Hoboken, NJ, pp81-96, 2004. 